\title{
La Formación Militar en la Escuela Técnica de la Fuerza Aérea Ecuatoriana desde el enfoque de la Modalidad Dual: Caso Tecnología Superior en Ciencias Militares Aeronáuticas
}

\section{The Military Training in the Technical School of the Ecuadorian Air Force from the Dual Modality approach - The case of Superior Technology in Aeronautical Military Sciences}

\author{
Jorge Pacheco Cabrera ${ }^{(1,2)}$, Edison Acurio Armas ${ }^{(1,2)}$, José Trujillo Jaramillo ${ }^{(1,2)}$ \\ ${ }^{(1)}$ Escuela Técnica de la Fuerza Aérea, Latacunga, Ecuador \\ (2) Tecnología Superior en Ciencias Militares Aeronáuticas \\ Universidad de las Fuerzas Armadas - ESPE, Latacunga, Ecuador \\ jorgepacheco69@outlock.com, eracurio@espe.edu.ec, jgtrujillo1@espe.edu.ec
}

\begin{abstract}
Resumen
Este estudio tiene como objetivo establecer una visión global de cómo se está implementando la Modalidad dual, reconocida por el Sistema de Educación Superior del Ecuador, dentro de la formación militar de los Aspirantes a Tropa de la Escuela Técnica de la Fuerza Aérea. Los nuevos instrumentos para la implementación de la modalidad, tales como: el Plan Marco de Formación, Plan de Aprendizaje Práctico y de Rotación, Manual Operativo de implementación y Convenio entre la Fuerza Aérea Ecuatoriana y la Universidad de las Fuerzas Armadas - ESPE, deberán complementarse con los instrumentos y políticas emitidas por el Modelo Educativo de Fuerzas Armadas para poder consolidar el proceso de formación del futuro soldado de la Fuerza Aérea Ecuatoriana. La importancia de un diseño curricular coherente y pertinente, un adecuado entorno real del aprendizaje con docentes/tutores capacitados y experimentados, el aporte de una adecuada coordinación, comunicación y el uso eficiente de recursos entre la Universidad y de la Escuela Técnica de la Fuerza Aérea, establecen una base sólida para iniciar este proceso de formación.
\end{abstract}

Palabras Claves: Diseño Curricular, Entorno real de aprendizaje, Formación Militar, Modalidad Dual, Modelo Educativo de F.F.A.A.

\begin{abstract}
The current study aims to establish a global vision of how the dual-modality, recognized by the Ecuadorian Higher Education System, is being implemented within the military training of Aspirants to Troop of the Air Force Technical School. The new instruments for the implementation of the modality, such as the Training Framework Plan, the Practical Learning and Rotation Plan, the Operational Manual for implementation, and the Agreement between the Ecuadorian Air Force and the Universidad de las Fuerzas Armadas - ESPE, needs to be complemented with the instruments and policies issued by the Educational Model of the Armed Forces in order to consolidate the process of training the future soldier of the Ecuadorian Air Force. The importance of a coherent and pertinent curricular design, an adequate natural learning environment with trained and experienced teachers/tutors, the contribution of adequate coordination, communication, and efficient use of resources between the University and the Technical School of the Air Force, allows establishing a solid foundation In order to start such training process.
\end{abstract}

Keywords: Curricular design, Real learning environment., Dual Modality, Military Formation, F.F.A.A., Educational Model.

Fecha de Recepción: 10/06/2021 - Aceptado: 31/07/2021 - Publicado: 15/09/2021

ISSN: 2477-9253 - DOI: https://dx.doi.org/10.24133/RCSD.VOL06.N01.2021.05 


\section{Introducción}

La Carrera de Tecnología Superior en Ciencias Militares Aeronáuticas fue creada para brindar una formación de nivel tecnológico-superior a los Aspirantes a Tropa de la Escuela Técnica de la Fuerza Aérea (ETFA). Esta es reconocida en el Sistema de Educación Superior a través de la Universidad de las Fuerzas Armadas - ESPE. Fue aprobada bajo la Modalidad de estudios dual por el Consejo de Educación Superior, el 03 de octubre de 2018, mediante Resolución RPC-SO-36-No.610-2018.

Esta carrera está centrada en la formación académica técnica-militar, orientada al desarrollo de competencias referidas al perfil profesional del soldado aerotécnico, acorde a su realidad, lo cual constituye un proceso eficiente de transferencia de conocimientos, habilidades, destrezas y valores, en el que el proceso de enseñanza aprendizaje mediante la modalidad dual, alcanza cada vez más niveles de relación teoría-práctica, dando lugar a una formación integral. Lo último es norma esencial a lo largo de su carrera militar.

Dentro de un estudio de pertinencia adecuado, se han considerado las tendencias y tensiones de la profesión aeronáutica y militar, en la cual se asume el compromiso y responsabilidad de preparar a los alumnos militares en formación, con sólidos conocimientos y capacidades relacionadas con la ejecución de operaciones militares y técnicas aeronáuticas, en apoyo a la Seguridad Integral y en cumplimiento de la misión de la Fuerza Aérea Ecuatoriana en lo referente a la Vigilancia y Control del espacio y soberanía aérea. Posee tres itinerarios académicos: Mantenimiento de Aeronaves, Apoyo a las Operaciones Aéreas y Seguridad en donde se agrupan 28 de las especialidades que tiene la Fuerza Aérea Ecuatoriana para su personal de Aerotécnicos conforme al Plan de Carrera que tiene la institución militar (ver Figura 1).

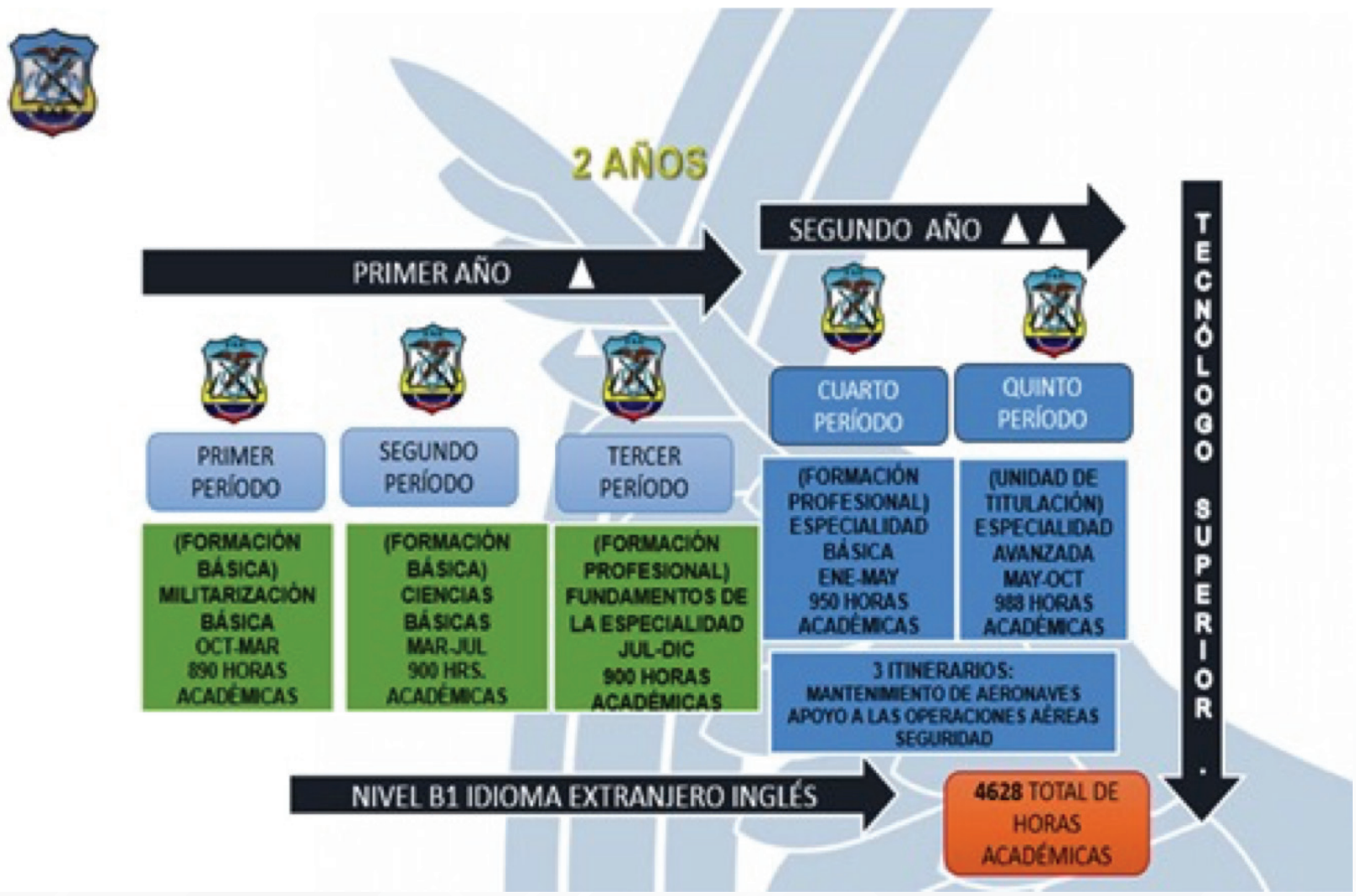

Figura 1. Estructura Curricular de la Tecnología Superior en Ciencias Militares Aeronáuticas. 
Su diseño curricular contempla 30 asignaturas divididas en 5 períodos académicos y tres campos de formación con 4628 horas, incluidas las prácticas pre-profesionales, las actividades de vinculación con la sociedad y la opción de titulación. Además, los resultados del aprendizaje de cada una de las asignaturas aportan a las competencias mínimas requeridas para la formación del personal militar, que están establecidas en el Modelo Educativo de las Fuerzas Armadas.

Las Fuerzas Armadas y sus instituciones educativas se encuentran metodológicamente direccionadas por un Perfil Genérico, al que se lo considera como un "conjunto de competencias genéricas y específicas que caracterizan al profesional militar para satisfacer las demandas institucionales y de la sociedad, que definen su personalidad y se desarrollan en el ejercicio de los cargos asignados de acuerdo con su grado y especialidad" (Comando Conjunto de las Fuerzas Armadas, 2012).

En el contexto de la formación tecnológica bajo el sistema de modalidad dual, el aprendizaje teórico práctico se desarrolla en un entorno educativo institucional bajo la responsabilidad de la Universidad de las Fuerzas Armadas - ESPE; en tanto que en los entornos laborales reales se desarrollarán fundamentalmente los componentes de aprendizaje práctico o de aplicación y experimentación de aprendizajes previamente adquiridos en el entorno institucional de la Fuerza Aérea Ecuatoriana y, en especial, dentro de su Escuela de Formación de personal de tropa, la ETFA.

\subsection{Competencias en el Sistema Educativo Militar Ecuatoriano:}

El perfil profesional del militar aerotécnico de la FAE está compuesto por un conjunto de competencias que debe haber desarrollado al graduarse de la Carrera de Tecnología Superior en Ciencias Militares Aeronáuticas y que, a su vez, podrá aplicar durante toda su carrera profesional de manera satisfactoria. Todo lo anterior lo hará capaz de desarrollar un aprendizaje permanente a lo largo de la vida.

Las competencias profesionales planteadas por el Modelo Educativo de las Fuerzas Armadas son de cuatro tipos: competencias básicas, competencias genéricas, competencias específicas y competencias transversales (ver Figura 2).

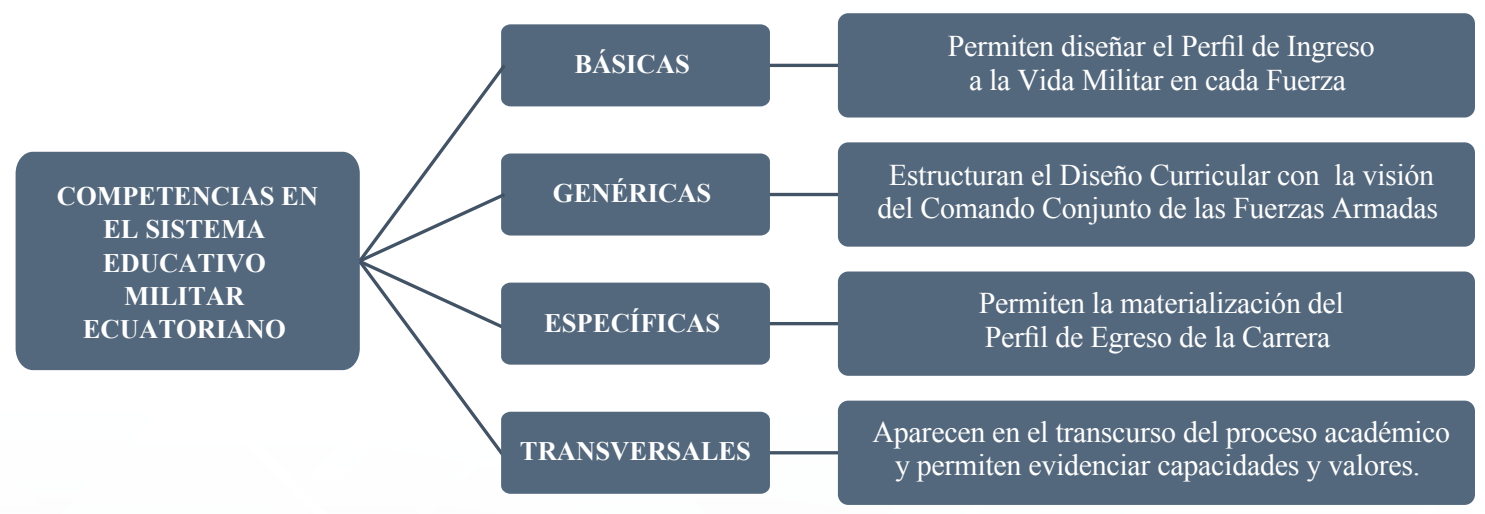

Figura 2. Tipos de competencias en el Sistema Educativo Militar Ecuatoriano.

Las competencias básicas son aquellas que debe haber desarrollado un joven al finalizar la enseñanza obligatoria para poder lograr su realización personal, ejercer la ciudadanía activa, incorporarse a la vida adulta de manera satisfactoria y ser capaz de desarrollar un aprendizaje permanente a lo largo de la vida (Bolívar \& Guarro, 2007). Estas competencias constituyen una parte del perfil de ingreso a los institutos de educación militar de formación (Arma y Servicios). 
Las competencias genéricas identifican los elementos compartidos que pueden ser comunes al ámbito de desempeño, cargos, funciones o tareas que cumplen los miembros de las Fuerzas Armadas en sus respectivos grados. Presentan una proyección generalizadora para el desempeño profesional durante toda la carrera militar en la sociedad, constituyen capacidades que posee el militar y que le permiten cumplir la misión constitucional. (Modelo Educativo de las Fuerzas Armadas, 2012). Son los conocimientos, habilidades, destrezas y valores que debe desarrollar el militar, de acuerdo con su grado y especialidad, para cumplir actividades y tareas de su campo ocupacional. Dentro de este campo de competencias, se encuentran la ejecución de conocimientos, habilidades y destrezas inherentes al correcto desempeño de las funciones de:

- Ayudante de una escuadrilla técnica o administrativo de una Sección Logística.

- $\quad$ Clase de Semana

- $\quad$ Fusilero en un Pelotón de Guardia

- Miembro de una Patrulla en Operaciones tipo Policía o en Operaciones Militares de Defensa Interna

- Miembro de un Grupo de Tarea o de Ayuda Comunitaria

- $\quad$ Miembro de Misiones de Mantenimiento de Paz.

Las competencias específicas operacionalizan el perfil profesional para cada grado y especialidad en cada uno de los Institutos de Educación Militar de las Fuerzas Armadas, a través de los diseños curriculares elaborados para los procesos de formación, perfeccionamiento, especialización y capacitación. (Modelo Educativo de las Fuerzas Armadas, 2012)

El desarrollo de estas competencias es secuencial, se encuentran en función del Plan de Carrera de la Fuerza Aérea y constituye el conjunto de conocimientos, habilidades y destrezas requeridas por el profesional graduado para aplicarlas, actualizarlas, perfeccionarlas y especializarlas a través de los años que dure en la carrera Militar.

Competencias técnicas aplicadas a la aeronáutica: de acuerdo con el grado y el nivel de formación Militar, las competencias específicas se van diversificando y orientando hacia campos técnicos puntuales de las Ciencias Aeronáuticas. Le corresponde al militar Tecnólogo Superior en Ciencias Militares Aeronáuticas el desarrollo de las siguientes competencias específicas:

- Aplica conocimientos relacionados a la Aeronáutica.

- Utiliza materiales propios de los sistemas aeronáuticos, de acuerdo con sus propiedades.

- Aplica procesos de manufactura utilizados en los sistemas y plataformas aeroespaciales.

- Emplea procesos de manufactura básica y de alta tecnología utilizados en aeronáutica.

- Maneja manuales y documentación técnica de aviación

- Lee e interpreta planos y diagramas técnicos para el Mantenimiento del poder Aéreo de la FAE.

- Repara daños detectados en los sistemas de la aeronave y potencial aéreo de la FAE 
- Maneja herramientas especiales y bancos de pruebas operacionales de los componentes y sistemas aeronáuticos.

- Emplea procedimientos de seguridad operacional en las actividades de carácter técnico relacionado con el campo aeronáutico.

- Resguarda la integridad humana en todo momento, minimizando el riesgo en las actividades que desempeña en su campo laboral.

- Aplica regulaciones y procedimientos, de acuerdo con estándares nacionales e internacionales de aeronavegabilidad.

- Administra recurso material y tecnológico, fundamentado su accionar en conocimientos de investigación aplicada.

Las competencias transversales permiten al militar maximizar su desempeño laboral y permiten la transformación del conocimiento en comportamiento adecuado. Este último es influido por habilidades cognitivas, principios, valores y actitudes. Son aquellas competencias las que, durante el proceso académico y formativo, permitirán evidenciar capacidades, actitudes y valores.

La Escuela Técnica de la Fuerza Aérea es parte del Sistema de Educación Militar de las Fuerzas Armadas. Tiene como finalidad formar, perfeccionar, especializar y capacitar permanentemente al personal militar, a fin de dotarlo de las competencias necesarias para el cumplimiento de los cargos, funciones y tareas previstos en la estructura institucional.

La Ley de Personal de las Fuerzas Armadas, por su parte, en su Art. 52 señala: "El Reclutamiento y Formación son el conjunto de actividades mediante las cuales se clasifica, ingresa e instruye a los ciudadanos ecuatorianos, para que presten sus servicios en las Fuerzas Armadas Permanentes y en las reservas, desde el llamamiento hasta su egreso de los centros de formación Militar". En los cursos de formación, donde en coordinación con las Instituciones de Educación Superior (IES) se otorguen títulos de nivel técnico superior, tecnológico superior y de tercer nivel o de grado, se deberá armonizar su desarrollo con las normas vigentes de la Educación Superior. (Comando Conjunto de las Fuerzas Armadas, 2009).

Con esta finalidad el Comando Conjunto de Fuerzas Armadas diseña el Modelo Educativo, que constituye el referente teórico, legal, metodológico y pedagógico de la forma como las Instituciones educativas que la conforman pueden operacionalizar la Misión constitucional. Corresponde a la Escuela Técnica de la Fuerza Aérea, a través de la Tecnología Superior en Ciencias Militares Aeronáuticas, cumplir con este mandato, creando la carrera que tendrá una duración de 5 ciclos académicos. Cada ciclo académico estará planificado en 18 semanas, brindando un total de 4628 horas para toda su formación profesional, incluyendo las 240 horas están dedicadas al desarrollo del Trabajo de Titulación, de acuerdo con lo establecido en la LOES.

\subsection{Campos de Formación}

El Modelo Educativo de las FF. AA considera a los ejes de formación como acciones que se deben desarrollar para abordar contenidos curriculares, que permitan desarrollar competencias para su perfeccionamiento, especialización, capacitación y la formación del personal militar especialista. El Sistema de Educación Militar de las Fuerzas Armadas contempla cinco ejes de formación militar 
de los cuales cuatro son considerados para el Diseño curricular de la Carrera, que al momento de diseñar los currículos se transforman en ejes curriculares, que se definen a continuación.

- $\quad$ Eje de Fundamentos Teóricos

- $\quad$ Eje de Ciencias Militares

- $\quad$ Eje de Cultura Militar

- $\quad$ Eje de Instrucción y Adiestramiento Militar

El eje de Ciencias Militares "son conocimientos, habilidades y destrezas que forman al profesional militar, se circunscriben a la formación, perfeccionamiento, especialización y capacitación para la seguridad-defensa y apoyo complementario a la seguridad integral del país. Corresponde a este eje, el horizonte epistemológico de seguridad y defensa con sus asignaturas o sus equivalentes, que dan sustento a la doctrina militar en vigencia en cada una de las Fuerzas" (Modelo Educativo de Fuerzas Armadas, 2016)

El eje de Cultura Militar "son conocimientos, habilidades y destrezas que refuerzan la carrera militar, son complementarios a los conocimientos del eje de las ciencias militares y se relacionan con ideas, tradiciones y costumbres que caracterizan a un militar, a una clase social, a una época histórica, etc.” (Modelo Educativo de Fuerzas Armadas, 2016)

El eje de Fundamentos Teóricos son todos los conocimientos y teorías que coadyuvan a la comprensión y contextualización de las problemáticas centrales de la carrera, y sus metodologías técnicas e instrumentos profesionales. En este campo se integran las asignaturas, cursos o sus equivalentes, que dan lugar a la articulación de la teoría y la práctica pre-profesional.

Finalmente, el eje de Instrucción y Entrenamiento Militar son todos los conocimientos, habilidades, destrezas y actividades extracurriculares que permiten el desempeño diario del Aspirante a Soldado, a través del Régimen Interno Militar, durante toda su permanencia en la Escuela Técnica de la Fuerza Aérea, fuera del Régimen Académico establecido en la malla curricular de la carrera.

Este último eje de Formación Militar no registra horas académicas en la carrera de Tecnología Superior en Ciencias Militares Aeronáuticas; sin embargo, este es de suma importancia para la convivencia interna y formación propia del militar, mediante el cumplimiento de disposiciones, normativas, procedimientos y actividades de Régimen Interno de la vida militar durante su período de formación, incluido el Idioma Inglés (Nivel B1) como requisito de graduación.

Este eje de formación militar se implementa a través del régimen interno, que está presente durante todo el proceso desde el ingreso hasta la graduación. Está basado en el cumplimiento de todas las actividades que están fuera de la carga horaria académica, en el cual los aspirantes a Soldados ejecutan la estricta observancia de la disciplina y subordinación en función del grado que ostentan y que les permite desarrollar el liderazgo y la conducta militar en el nivel que les corresponde. Algunas de las actividades del régimen interno militar son:

- Horario común para levantarse (Diana)

- Horario para descanso (Parte de Retreta) 
- Horarios para el Rancho (Alimentación)

- Revista de villa (Habitaciones)

- Revista de Armas

- Revista de aseo

- $\quad$ Parte de izada y arriada de la Bandera

- Guardia en las Instalaciones fuera del horario académico

- Parte y relevos de la guardia

- Imaginaria, guardia diurna y nocturna

- Horario de franquicia

- Aseo y limpieza de instalaciones

- Mantenimiento del armamento

- Cultura Física

- $\quad$ Estudios de Inglés (Nivel B1)

- $\quad$ Biblioteca (Estudios y Tareas)

- Relación de disciplina

- Repasos para ceremonias militares y desfiles

- Marchas administrativas

- Funciones de Alumno de Servicio, Semana y cuartelero

- Otros.

El horario de clase (Régimen Académico) está comprendido de lunes a viernes de 7:00 de la mañana a 13:30 y en la tarde de 14:30 a 16:30. El resto del día y tiempo le corresponde la formación militar propiamente dicha mediante el cumplimiento de Régimen Interno de Disciplina Militar, ejecutado durante todo el día y todos los días, incluidos sábados y domingos, puesto que la Formación Militar se desarrolla con régimen de internado.

Los ejes de Formación militar se relacionan con las Unidades de Organización Curricular para dar una estructura coherente que, considerando la transversalidad, permita el diseño de una malla curricular pertinente y adecuada con las necesidades de la sociedad ecuatoriana y de las Fuerzas Armadas.

La Formación dual es una modalidad, ya contrastada en otros países, de oferta académica y formativa localizada fundamentalmente dentro del ámbito de la formación profesional. Se caracteriza por la alternancia combinada de los procesos de enseñanza y aprendizaje en la empresa $\mathrm{y}$ en el centro de formación. Permite al alumno tener un contacto real con el trabajo, obteniendo experiencia y competitividad profesional y una mayor integración entre teoría y práctica, al no ser el centro docente la única fuente de conocimiento, transformando así el aprendizaje en un modelo dinámico y versátil. De esta forma, el aprendiz recibe orientaciones del tutor académico y del instructor de la empresa asociada, con la finalidad de efectuar labores prácticas en las que pueda aplicar de forma directa los conocimientos teóricos adquiridos, a su vez, en el centro educativo. Además, permite el acceso a estudios superiores con posterioridad, convirtiéndose en un sistema educativo permeable y dúctil. (Cámaras de Comercio España, Ministerio de Educación, Cultura y Deporte y Fondo Social Europeo, 2012). 
El origen de esta modalidad se remonta a la Alemania de finales de los años sesenta y principios de los setenta, específicamente en la región de Baden-Württemberg. Este país cuenta con gran experiencia y trayectoria en la implementación de la modalidad dual. Su planteamiento y desarrollo vino dado por dos factores: la masificación de las universidades tradicionales y la falta de mano de obra calificada, que era reclamada por las empresas en función de las nuevas realidades profesionales y laborales de la época.

El Estado, las empresas y las organizaciones empresariales, gremiales y sindicatos, intervienen en este proceso de formación, en el cual juntos determinan los contenidos académicos de cada una de las profesiones que demanda el mercado para su posterior aprobación en los Ministerios de Educación y Economía.

El objetivo de la formación profesional en modalidad dual es proporcionar a la empresa formadora la planificación de los recursos humanos a corto, mediano y largo plazo, con la finalidad de disponer de un número considerable y suficiente de empleados capacitados, acorde a las nuevas exigencias del puesto de trabajo. De esta manera, se cubre la necesidad de contar con una mano de obra calificada.

El costo de la formación es una cuestión frecuentemente discutida. Si bien los costos y beneficios de la formación dual no se pueden calcular de una manera precisa, existen algunas directrices y consideraciones que permiten estimarlos de una forma muy aproximada. Los costos más representativos del proceso de formación dual podrían considerarse:

- Remuneración de la formación

- Seguridad Social

- Prestaciones sociales voluntarias y bonos

- Tasas y Aranceles

- Costos de formación adicional

- Costos de recursos de capacitación

- Costos del personal docente y formador

- Costos de equipos de seguridad

Dentro de los beneficios se pueden enunciar los siguientes:

- Prestaciones pagadas directamente por los clientes.

- Colaboración productiva en la empresa, que el cliente no abona directamente, pero que influye en los resultados de la empresa.

- Beneficios indirectos cuantificables, como por ejemplo cuando una empresa no tiene la política de brindar capacitación a sus empleados y necesita contratar un experto cualificado.

- Beneficios indirectos no cuantificables, al generar un mínimo riesgo de una contratación de personal errónea.

Todo este proceso de formación termina con la realización de un examen (práctico y teórico) que generalmente lo realizan las Cámaras de Industria y Comercio, que son las entidades responsables de emitir las acreditaciones para la obtención de las competencias profesionales.

En el Ecuador, la modalidad dual fue reconocida oficialmente como una modalidad de estudio o aprendizaje desde 2014 cuando en el Reglamento de Régimen Académico expedido por el CES, se la incluye como parte de la formación en el Sistema de Educación Superior. 
En el 2016, se expide una Normativa para Carreras y Programas en Modalidad Dual, hasta que en el año 2018 recién se aprueba el Reglamento para las Carreras y Programas en Modalidad de formación Dual. En este último se definen los actores del proceso, responsabilidades, estructuras académicas, pero, sobre todo, se establecen los instrumentos esenciales para la implementación de la modalidad, que son:

- Plan Marco de Formación

- Plan de aprendizaje Práctico y de Rotación

- Convenio para la modalidad dual

- Manual Operativo para la implementación de carreras y programas en modalidad de formación dual.

Una vez que se definen las políticas y el marco normativo en los cuales la carrera de Tecnología Superior en Ciencias Militares Aeronáuticas gestionará su funcionamiento bajo la modalidad dual, se verifica el cumplimiento de lo declarado en el diseño curricular aprobado por el CES con la primera cohorte de estudiantes. Esto se consigue a través de la revisión de los formatos descritos en el Plan Marco de Formación, Plan de aprendizaje práctico y Manual Operativo de la Carrera en formación dual.

\section{Materiales y Métodos}

Para poder lograr la implementación de la carrera de Tecnología Superior en Ciencias Militares Aeronáuticas en modalidad dual, se consideraron varios aspectos metodológicos a fin de conseguir resultados precisos sobre sus puntos negativos y positivos para su posible ejecución. Como primera fase, se recopiló información documental de políticas, lineamientos, normas, reglamentos y formatos de instrumentos curriculares otorgados por las diferentes autoridades, tanto en el sistema de educación superior como en el sistema de educación militar. Entre lo investigado hay materiales como: el reglamento de régimen académico, reglamento para las carreras y programas en modalidad de formación dual, reglamento de carrera y escalafón del profesor e investigador del sistema de educación superior, modelo educativo de Fuerzas Armadas, reglamento de educación militar, plan Marco de Formación de la Carrera, plan de aprendizaje práctico y de rotación y el manual de implementación de la modalidad dual. Todo lo recopilado tiene como objetivo analizar los aspectos principales exigidos para la aplicabilidad de la modalidad en cuestión.

En la segunda fase, se recopiló información del Departamento académico y de evaluación a fin de obtener datos del proceso o métodos de enseñanza de los últimos años.

En la tercera fase, se realizaron entrevistas al personal docente y administrativo para el análisis del grado de conocimiento sobre el proceso de formación la modalidad dual. Los resultados obtenidos fueron poco alentadores.

En la cuarta fase, se analizaron documentos curriculares de cumplimiento de la malla curricular, seguimiento al sílabo, portafolios docentes, evaluaciones docentes, horarios y formatos declarados en los instrumentos para la implementación de la modalidad dual en la carrera.

Como última fase, se realizó la observación directa de las instalaciones a fin de corroborar el estado de las infraestructuras, talleres, laboratorios, hangares y simuladores, proceso con el que, como ente formador, se debe cumplir según las exigencias del CES.

Por todos los aspectos señalados se considera a esta investigación de tipo documental, de campo y descriptiva. 


\section{Evaluación de Resultados y Discusión}

Una vez analizada toda la documentación, se determinó la necesidad de capacitar al personal docente y administrativo y de implementar laboratorios para meteorología y operaciones aéreas, talleres de motores, talleres de hélices, talleres de estructuras aerodinámicas, áreas para el desarrollo de defensa aérea e inteligencia, para que el estudiante pueda lograr un $70 \%$ de práctica en el ente formador. De esta manera, el estudiante una vez graduado puede cumplir funciones inherentes a la especialidad, tales como línea de vuelo o mantenimiento de campo en los hangares de la FAE.

Con estos resultados en la parte administrativa y bajo la estructura de una Unidad Académica Especial, se evaluó la corresponsabilidad existente en el proceso formativo entre la ESPE y la ETFA, principalmente en la asignación de recursos para potencializar la infraestructura académica como aulas, entornos virtuales, bibliotecas y laboratorios.

Ya cubiertas las necesidades administrativas y académicas para la implementación de la modalidad dual, se procede a su ejecución de la siguiente manera: el sistema tiene una duración de 2 años, distribuidos en 5 períodos académicos, durante los cuales el estudiante militar recibe una formación integral en dos escenarios de aprendizaje:

- En la Universidad de las Fuerzas Armadas - ESPE, en donde se adquieren los conocimientos de fundamentación teórica (aula) y prácticos (talleres y laboratorios).

- En la Escuela Técnica de la Fuerza Aérea y Repartos militares de la FAE, en donde los conocimientos adquiridos se integrarán a los procesos militares aeronáuticos que requiere la Fuerza Aérea Ecuatoriana.

Los escenarios de aprendizaje son los lugares físicos en los cuales los estudiantes militares desarrollarán aprendizajes que, partiendo de la teoría, les permitan alcanzar habilidades y destrezas requeridas por el perfil profesional. Cabe destacar que, por la naturaleza de ser una carrera innovadora y en modalidad dual, estos escenarios de aprendizaje corresponden a: Alas de Combate o Repartos Militares de la Fuerza Aérea Ecuatoriana distribuidos a nivel nacional (Ala11 Latacunga), (Ala 21 Taura), (Ala 22 Guayaquil), (Ala23 Manta), (ESMA - Escuela de Formación de Oficiales Salinas), además de laboratorios, hangares, centros de mantenimiento, aviones, infraestructura aeronáutica, etc. Al ser todo lo anterior propiedad de la Fuerza Aérea Ecuatoriana, es posible utilizarlas a través de la Escuela Técnica de la Fuerza Aérea, gracias a los convenios firmados entre la ESPE y la Entidad Formadora, como lo exige la modalidad dual (ver Figura 3).

Según el Art. 20 del Reglamento de Régimen Académico codificado, expedido por el Consejo de Educación Superior CES, "Las unidades de organización curricular son formas de ordenamiento de las asignaturas, cursos o sus equivalentes a lo largo de la carrera o programa, que permiten integrar el aprendizaje en cada período académico, articulando los conocimientos de modo progresivo". Por esta razón, la Tecnología Superior en Ciencias Militares Aeronáuticas ha sido estructurada bajo tres Unidades de Organización Curricular y estas son:

- Unidad básica (primer y segundo nivel)

- Unidad profesional (tercero y cuarto nivel)

- Unidad de Titulación (quinto nivel) 

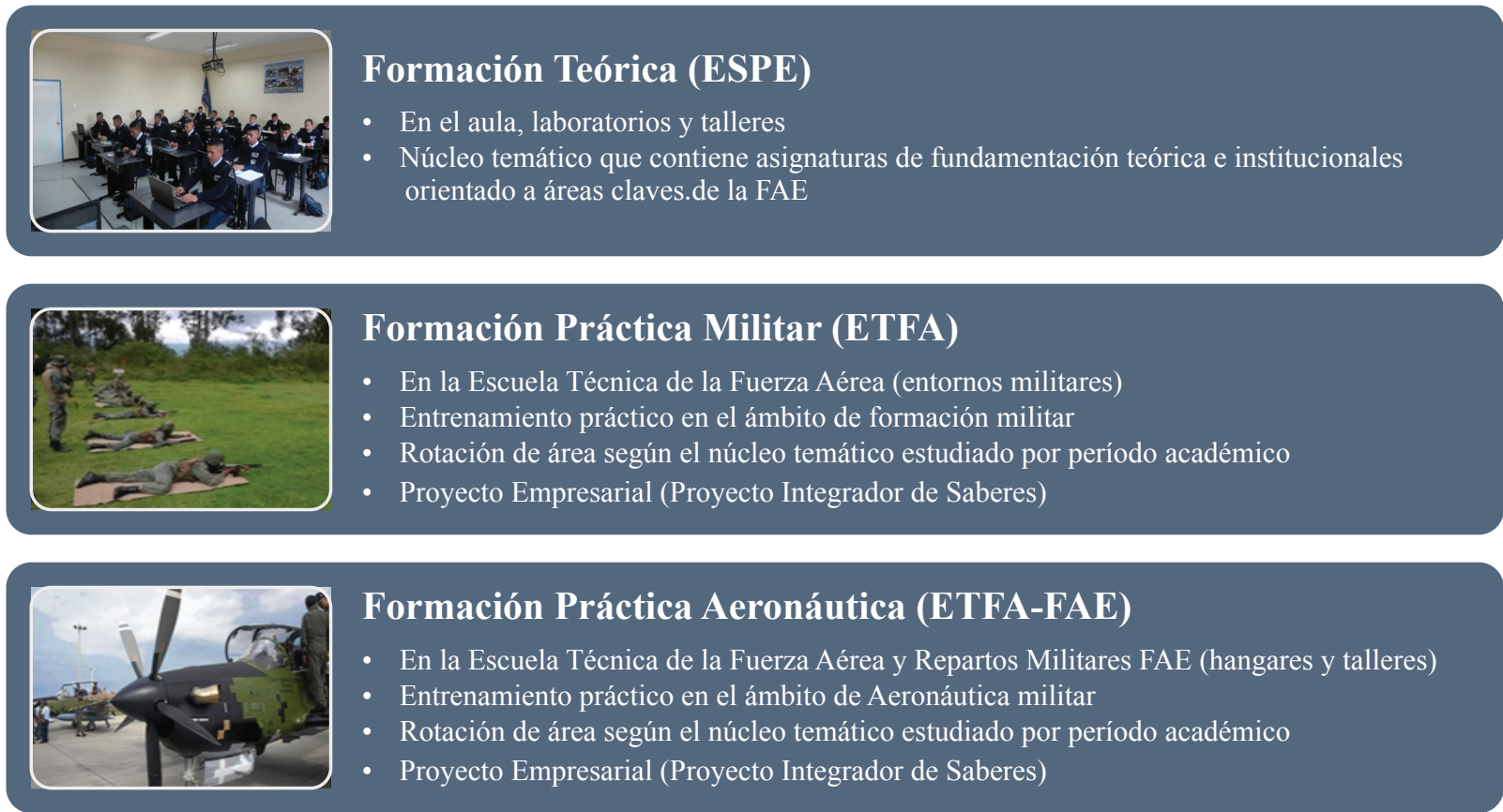

\section{Formación Práctica Aeronáutica (ETFA-FAE)}

- En la Escuela Técnica de la Fuerza Aérea y Repartos Militares FAE (hangares y talleres)

- Entrenamiento práctico en el ámbito de Aeronáutica militar

- Rotación de área según el núcleo temático estudiado por período académico

- Proyecto Empresarial (Proyecto Integrador de Saberes)

Figura 3: Escenarios de aprendizaje de la Carrera.

La Unidad Básica de formación es la unidad curricular que introduce al estudiante en el aprendizaje de las ciencias y disciplinas que sustentan la carrera, sus metodologías e instrumentos, así como en la contextualización de los estudios profesionales (Reglamento de Régimen Académico, 2012).

La carrera de Tecnología Superior en Ciencias Militares Aeronáuticas está planificada con 1790 horas, cuya finalidad es establecer los principios y pilares fundamentales de la carrera. Para ello se recurre a dos niveles de formación cuyo objeto de estudio son los principios básicos y fundamentales de la formación militar y aeronáutica.

Tomando como referencia la primera cohorte de Tecnólogos de la carrera, donde ingresaron 195 estudiantes, en el primer período se desarrolló el Proyecto integrador "Contextos militares y aprendizaje de las Plataformas para la vigilancia y control del Aero espacio en cumplimiento de la misión de la Fuerza Aérea Ecuatoriana", con el aporte de las asignaturas: Instrucción formal y Ceremonial Militar, Organización y técnicas para un combatiente individual, Conocimiento de armas y Plataformas para la Vigilancia y Control del espacio aéreo, Historia de la aviación y doctrina aeroespacial; Normativa Militar y Comunicación Oral y Escrita. Estas asignaturas persiguen transformar y orientar al estudiante a la vida militar y su vinculación directa con la Fuerza Aérea Ecuatoriana.

Para la evaluación de esta primera unidad de organización, se recurrió a la presentación de una formación en escuadrilla militar de fusileros, formación de patrullas, el porte militar que posee el estudiante y un ensayo sobre Lineamientos importantes de la doctrina Aeroespacial de la FAE y su potencial aéreo para el cumplimiento de su misión.

Para el segundo período se desarrolló el Proyecto Integrador "Aproximación de los fundamentos técnicos de Aeronáutica y aplicación de una cultura de Seguridad Operacional”, que se gestionó con el aporte de las siguientes asignaturas: Operaciones militares para la Defensa Interna, Ciencias Aplicadas a 
la Aeronáutica, Materiales y Procesos de Manufactura, Matemática Aplicada a la Aeronáutica, Seguridad Operacional y Actuación Humana y Herramientas ofimáticas de aplicación profesional.

La evaluación de este segundo período de formación se realizó a través de la construcción de una pequeña aplicación aeronáutica. Esta actividad contribuirá en la formación del profesional para identificar, analizar y diagnosticar materiales, fundamentos básicos y seguridad operacional utilizados en las plataformas aeronáuticas.

La Unidad Profesional es la unidad curricular que está orientada al conocimiento del campo de estudio y las áreas de actuación de la carrera, a través de la integración de las teorías correspondientes y de la práctica pre profesional. (Reglamento de Régimen Académico, 2012).

La carrera está constituida por 1850 horas, en las que se contemplan 160 horas de prácticas por vinculación con la sociedad. Esta unidad se conforma por dos niveles de formación, en los que se desarrollan las capacidades específicas de la profesión que fortalecen al estudiante con su formación técnica, fusionando la teoría con la práctica en los talleres y laboratorios especializados en el campo aeronáutico que posee la Escuela Técnica de la Fuerza Aérea.

En el tercer período se desarrolló el Proyecto Integrador "Contextos técnicos de aeronáutica y Mantenimiento del poder aéreo de la FAE".

Este proyecto se llevó a cabo con el aporte de las siguientes asignaturas: Organización y Técnicas para apoyo a la Seguridad Integral, Sistemas de propulsión aeronáutica y aeroespacial, Sistemas CADCAM, Física Aplicada a la Aeronáutica, Mercancías peligrosas, Fundamentos de las Ciencias Aeronáuticas y Programación y metodologías de sistemas.

Para la evaluación del tercer período de formación se realizó un proyecto de aplicación de procedimientos y normativas técnicas utilizadas en aeronáutica, lo que contribuirá en la formación del futuro profesional en el desarrollo de habilidades para utilizar manuales técnicos, herramientas, formularios, registros técnicos, diagramas y procedimientos utilizados en aeronáutica.

El cuarto período de formación contempla el desarrollo del Proyecto Integrador "Aplicación y evaluación de procedimientos técnicos aplicados en el mantenimiento del poder aéreo que posee la FAE". Las asignaturas que contribuyen con la realización de esta actividad son: Estructuras de aeronaves, Apoyo a las operaciones aéreas, Metodología de la Investigación Científica, Liderazgo, Idioma extranjero técnico y Especialidad Básica (en cada uno de los itinerarios académicos propuestos).

Este período de formación se evaluó mediante la realización de un proyecto o estudio de caso que integre el funcionamiento global de los sistemas que comprenden las plataformas aéreas y solucione un problema supuesto que tenga el equipo o aeronave del poder aéreo que posee la FAE.

La Unidad de titulación es la unidad curricular que incluye las asignaturas, cursos o sus equivalentes, que permiten la validación académica de los conocimientos, habilidades y desempeños adquiridos en la carrera para la resolución de problemas, dilemas o desafíos de una profesión. Su resultado final fundamental es: a) el desarrollo de un trabajo de titulación, basado en procesos de investigación e 
intervención o, b) la preparación y aprobación de un examen de grado de carácter complexivo (Reglamento de Régimen Académico, 2012).

Esta unidad está constituida por 988 horas de trabajo, que se desarrollan en el último período de formación y persiguen validar las competencias profesionales adquiridas mediante el desarrollo de un trabajo de titulación que garantiza el logro máximo de los saberes para su formación militar y técnica aeronáutica. En este período se construye el proyecto de integración de saberes "Opción de Titulación: Aplicación de estándares, procedimientos y normativas técnicas en procedimientos militares y aeronáuticos". Este trabajo final será la recopilación de todas las áreas vistas en la carrera, adicionando las materias Sistemas Auxiliares, Cultura Ambiental, Realidad Nacional y Geopolítica, Derecho aplicado a las operaciones militares y aeronáuticas y Especialidad Avanzada.

La evaluación integral y total garantiza la consolidación del conocimiento adquirido durante todo el proceso de aprendizaje, reflejado en su trabajo de titulación. Además, el nivel de deserción fue del $6.66 \%$ para la primera cohorte, en la que se graduaron 182 soldados como tecnólogos en Ciencias Militares Aeronáuticas.

Las formas de organización de los conocimientos en función de sus propósitos y objetivos son conocidos como campos de formación, los que para esta carrera se han aplicado respetando la naturaleza de la formación militar y aeronáutica; es decir, avocando los contenidos propios de la ciencia y cultura militar dentro del proceso de formación (ver Figura 4).

\section{TECNÓLOGO SUPERIOR EN CIENCIAS MILITARES}

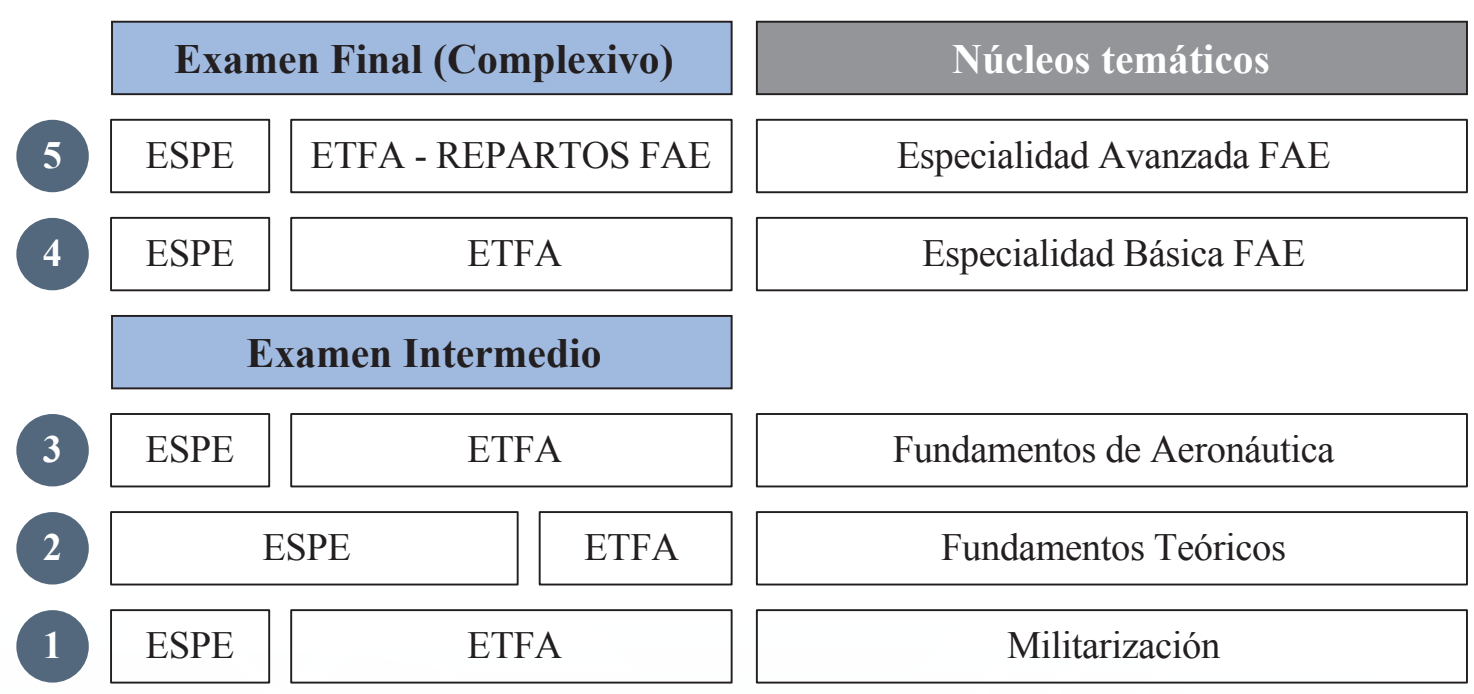

Figura 4: Estructuración de la Carrera según núcleos temáticos

La implementación, ejecución y viabilización de este currículo se materializó gracias a los instrumentos para la Implementación de la modalidad dual, que fueron declarados y aprobados en el Consejo de Educación Superior - CES (ver Figura 5). 
A diferencia de la modalidad presencial o tradicional, la modalidad dual generó una ventaja competitiva de relevancia, ya que mejora en cierta manera el proceso de crecimiento de competencias laborales del alumno militar, optimizando los recursos, pero, sobre todo, asegurando la cualificación laboral del estudiante en el entorno laboral real dentro de la Fuerza Aérea Ecuatoriana.

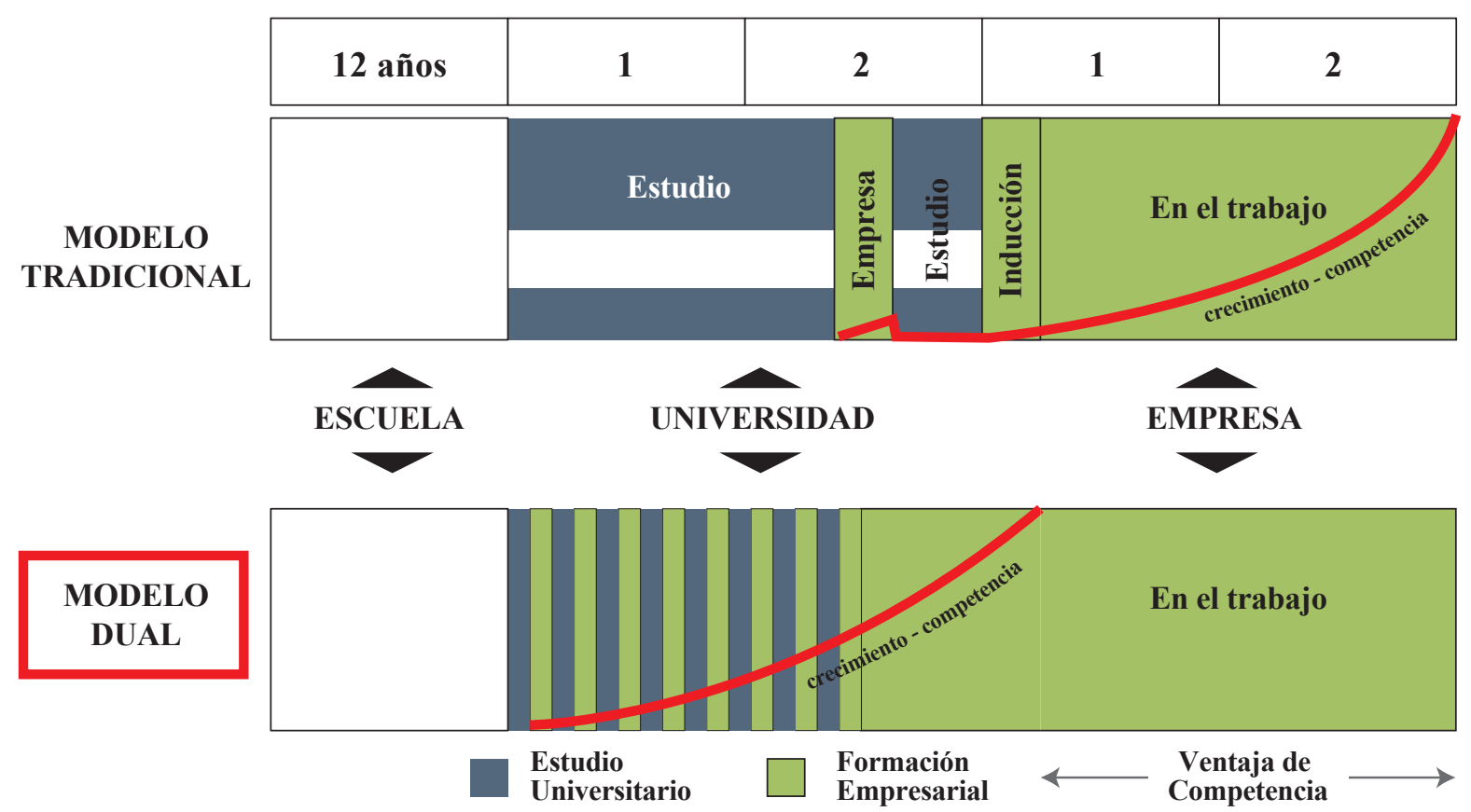

Figura 5: Ventajas de la modalidad dual respecto al modelo tradicional

Dentro de todo este proceso, tanto la Universidad de las Fuerzas Armadas - ESPE como la Escuela Técnica de la Fuerza Aérea priorizaron la Seguridad del estudiante. Es por esta razón que se dotó de equipo de protección personal y seguro de accidentes para cada una de las actividades que realizan los aspirantes a tropa dentro de su formación. Esto permitió que durante el proceso académico no existieran accidentes de ningún tipo.

Los laboratorios e infraestructura académica que posee la Escuela Técnica de la Fuerza Aérea cumplen los estándares para realizar un proceso de formación adecuada. Las aulas tienen un área de $49 \mathrm{~m} 2$ y tiene capacidad para 25 estudiantes.

Cuenta con espacios para el aprendizaje práctico, tanto en la parte militar como en la aeronáutica, muy amplio, debidamente señalizado, iluminado y con suficiente ventilación. Todo lo anterior se gestiona con procesos que emplean formatos para uso, calibración, mantenimiento de los equipos y maquinaria. Falta por implementar los laboratorios de Física Básica, Materiales y Procesos de Manufactura, Mercancías peligrosas, Computación e inglés para la suficiencia del idioma extranjero, que son responsabilidad de la Universidad de las Fuerzas Armadas - ESPE.

En la Escuela Técnica de la Fuerza Aérea, el formador o tutor específico es aquella persona responsable de la formación, quien debe estar calificado personal y profesionalmente. Es el responsable de planificar, ejecutar y controlar la formación profesional conforme al Plan marco de formación, Plan de aprendizaje práctico y Plan de rotación. 

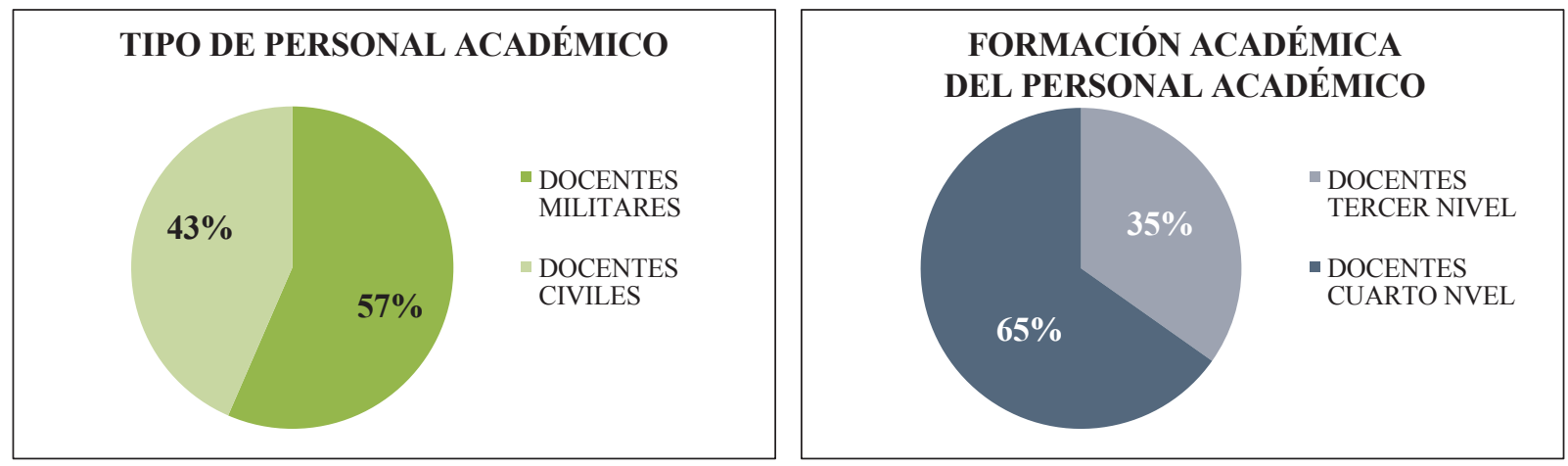

Figura 6 Personal Académico (Docentes/Tutores) de la carrera para la primera cohorte.

El personal docente cuenta con la formación académica y experiencia pertinente a la asignatura designada. Sus características y habilidades como formador son: especialista y experto en el área aeronáutica y militar, pedagogo y mentor, jefe, modelo y empleado directo de la FAE. El oficial designado como tutor específico tiene dentro de su formación académica una maestría en educación y mucha experiencia en el proceso formativo militar dentro de la FAE.

La capacitación personal y técnica del formador son requisitos mínimos legales, cuyos criterios están claramente definidos y son demostrables. Ocupa una posición clave en la ETFA y FAE. Prácticamente el 90\% del personal académico de la ETFA posee al menos una cualificación profesional de Formador de Formadores, Instructor OACI-TRAINAIR y Formador de Formadores Internacional avalado por la Cámara de Comercio Ecuatoriano-Alemana (ver Figura 6).

Como multiplicador de conocimiento y consejero educativo, no solo fomenta en los aspirantes a tropa las competencias técnicas, sino también la disposición y capacidad para el trabajo en equipo, la consciencia de responsabilidad, la disposición a la co-creación y la identificación institucional con la FAE.

Finalmente, una vez creado el entorno adecuado para el proceso de formación, asignados los recursos por parte de la Universidad y la FAE, planta de docentes/tutores y cumpliendo con lo establecido en los Instrumentos para la Implementación de la modalidad y diseño Curricular aprobado, se esperan los siguientes beneficios a corto y largo plazo:

En el Proceso de Reclutamiento

- Reducción de costos de inducción y capacitación en la Carrera militar-aeronáutica

- Disminución de rotación

- Minimiza el riesgo de contratación de personal con un perfil no adecuado.

- La ETFA conoce y forma las competencias del futuro Aerotécnico en base al Plan de Carrera FAE.

En el Perfil Profesional

- Malla curricular ajustada a la realidad productiva de la Fuerza Aérea

- Actualización de núcleos temáticos de acuerdo a la necesidad de la FAE

- Formación práctica dentro de su propio entorno en base al Poder Aéreo que dispone la FAE. 
En la Productividad

- El aspirante a tropa adquiere su experiencia específica durante su formación.

- Conocimiento de la cultura organizacional y los procesos logísticos y de mantenimiento de la FAE

- El aspirante realiza su trabajo de titulación y proyectos que responden a la necesidad institucional de la FAE.

- Organiza la producción de servicios de seguridad de una manera técnica, de acuerdo a sus conocimientos teóricos y la experiencia en la FAE.

- Un equipo formado adecuadamente conlleva a entregar productos y servicios de calidad, mayor productividad y competitividad, a optimizar tiempo, recursos y materias primas.

En la Responsabilidad social y con el país

- La FAE a través de la Escuela Técnica de la Fuerza Aérea puede incorporar al Aspirante a tropa a la estrategia de responsabilidad social corporativa y el compromiso de servir a la Patria desde su puesto laboral.

\section{Conclusiones y Trabajo Futuro}

De este estudio se obtienen las siguientes conclusiones:

- La modalidad dual en la formación militar permite obtener el máximo rendimiento de la formación impartida para el aspirante a tropa. Esto teniendo en cuenta que el mejor escenario para ese fin es el establecido en el entorno laboral real dentro del campo militar y aeronáutico en la Fuerza Aérea Ecuatoriana, priorizando el componente práctico para la obtención de las competencias declaradas en el sistema educativo militar ecuatoriano.

- Los instrumentos para la implementación de la modalidad dual (Plan Marco de Formación, Plan de aprendizaje práctico y de rotación y el Manual operativo para la implementación) otorgan las directrices, políticas y responsabilidades claras a cada uno de los actores principales del sistema de formación.

- La formación militar que recibe el aspirante a tropa o Aerotécnico avanzará en forma paralela y coordinada con la ejecución de la Tecnología Superior en Ciencias Militares Aeronáuticas, de tal manera que los cinco ciclos académicos que durará la tecnología, estarán dentro de los dos años que toma su formación militar. Este escenario mejora en cierta manera el proceso de crecimiento de competencias laborales del alumno militar, optimizando los recursos, pero, sobre todo, asegurando la cualificación laboral del estudiante en el entorno laboral real dentro de la Fuerza Aérea Ecuatoriana.

- La experiencia, integridad y formación de los docentes/tutores académicos en la carrera no solo motivan y fomentan en los aspirantes a tropa las competencias técnicas, sino también la disposición y capacidad para el trabajo en equipo, la consciencia de responsabilidad, la disposición a la cocreación y la identificación institucional con la FAE.

- La Universidad de las Fuerzas Armadas - ESPE y la Escuela Técnica de la Fuerza Aérea deben garantizar las condiciones adecuadas de entornos de aprendizaje en un ambiente de seguridad, calidad e practicidad de la formación del aspirante a tropa. 


\section{Referencias Bibliográficas}

Cámara Ecuatoriana-Alemana de Comercio e Industria, (2018), Texto de Curso Formador de Formadores, Quito, Ecuador AHK.

Cámaras de Comercio, Ministerio de Educación, Cultura y Deporte y Fondo Social Europeo, (2015), Guía de Formación Dual, España, Autor.

Consejo de Educación Superior, (2019), Reglamento de Régimen Académico, recuperado de http://www. ces.gob.ec

Consejo de Educación Superior, (2018), Reglamento para las carreras y Programas en Modalidad de formación dual, recuperado de http://www.ces.gob.ec

Comando Conjunto de las Fuerzas Armadas, (2012/2016/2018), Modelo Educativo de las Fuerzas Armadas, Quito, Ecuador, Medios Públicos EP.

Dale Crane, (2011), Aviation Maintenance Technician Series: General, Airframe, Power Plant, Washington, USA, Aviation Supplies \& Academics, Inc.

Dale Crane, (2012), Aviation Mechanic Handbook, Washington, USA, Aviation Supplies \& Academics, Inc.

Dirección General de Aviación Civil del Ecuador, (2016), Recopilación de Derecho Aéreo- TOMO VI (parte 147), Quito, Ecuador, Corporación de Estudios y Publicaciones.

Federal Aviation Administration, (2003), Aviation Mechanic General, Washington, USA, ASA.

Fuerza Aérea Ecuatoriana, (2010), Doctrina Aeroespacial Básica, Quito, Ecuador, Autor.

Instituto Español de Estudios Estratégicos, (2013), América Latina: Desafíos para la integración en Seguridad y Defensa, recuperado de http://www.ieee.es

Ministerio de Coordinación de Seguridad, (2011), Plan Nacional de Seguridad Integral, Quito, Ecuador, Manthra Editores.

Ministerio de Defensa Nacional, (2014), Agenda Política de la Defensa 2014-2017, Quito, Ecuador, Autor.

Ministerio de Defensa Nacional del Ecuador, (2014), Agenda de Investigación, Desarrollo Tecnológico e Innovación para el Sector Defensa 2014-2017, Quito, Ecuador, Autor

Ministerio de Defensa Nacional, (2012), Requisitos para el Ingreso a las Escuelas Militares, Quito, Ecuador, Autor.

Ministerio de Defensa Nacional del Ecuador - IAEN, (Agosto, 2014), Retos de la Educación Militar y de la Formación en Defensa para el Buen Vivir, Seminario-Taller Internacional, Taller dirigido por Ministerio de Defensa Nacional del Ecuador - IAEN, Quito

Reithmaier Larry, (1999), Standard Aircraft Handbook for Mechanics and Technicians, Washington, USA, Mc. Graw Hill.

Secretaría Nacional de Planificación y Desarrollo - SENPLADES, (2017), Plan Nacional de Desarrollo / Plan Nacional para el Desarrollo Toda una Vida 2017-2021, recuperado de http://www.planificación. gob.ec 